\title{
Relação do nível de atividade física habitual e consumo de medicamentos em usuários do Sistema Único de Saúde
}

\author{
Relationship level of physical activity and drug consumption in the \\ unified health system users
}

Cledir Lago', Geiziane Laurindo de Morais', Flavio Renato Barros da Guarda², Gustavo de Oliveira³, Priscyla Waleska Simões ${ }^{4}$, Joni Marcio de Farias ${ }^{4}$

\section{Resumo}

O objetivo da pesquisa foi avaliar a relação entre o nível de atividade física habitual, o uso de medicamentos e as relações sociodemográficas na população pertencente ao Distrito Sanitário da Santa Luzia da cidade de Criciúma - SC. A população estudada foi composta de 386 indivíduos de ambos os gêneros, utilizando instrumentos padronizados por meio de uma pesquisa censitária e inquérito domiciliar. Os dados coletados foram informações pessoais (idade e Índice de Massa Corporal - IMC), nível de atividade física e classificação econômica, sendo analisados por meio do programa SPSS versão 20.0. Os resultados apontam maior participação nas entrevistas de mulheres (72\%) com maior prevalência no consumo de medicamento também pelas mulheres $54,66 \%$. Quanto a quantidade utilizada, o uso foi maior entre os homens 2,44 $( \pm 1,34)$. A amostra foi considerada inativa quanto ao nível de atividade física habitual e acima do peso, demonstrando relação entre consumo de medicamentos de acordo com a idade e IMC e estilo de vida. Com esses resultados pode-se concluir que o aumento do consumo de medicamentos apresenta relação com o envelhecimento, o aumento do IMC e redução da prática de atividade física, demonstrando a necessidade de implantação de programas para orientação e promoção da saúde por meio do exercício físico.

\section{Palavras-chave}

Atividade motora; Uso de medicamentos; Saúde Pública.

\begin{abstract}
The objective of the research was to evaluate the relationship between the level of habitual physical activity, use of medication and sociodemographic relations in the population belonging to the Sanitary District of the Santa Luzia city of Criciuma - SC. The study population consisted of 386 individuals of both genders, using standardized instruments through a census survey and household survey. Data were personal information (age and body mass index - BMI), physical activity level and economic status, and analyzed using SPSS version 20.0. The results show increased participation in women of interviews (72\%) with a bigher prevalence in drug consumption also by women $54.66 \%$. As for the amount used, the use was higher among men $2.44 \pm 1.34$. The sample was considered inactive in the level of habitual physical activity and overweight, showing relation between consumption of drugs according to age and BMI and lifestyle. With these results we can conclude that the increased consumption of drugs is correlated with age, increased BMI and reduced physical activity, demonstrating the need to implement programs for guidance and health promotion through physical exercise.
\end{abstract}

\section{Keywords}

Motor Activity; Drug Utilization; Public Health.

\section{Introdução}

O estilo de vida é considerado fundamental e determinante para o estado de saúde, principalmente quan-

1 Universidade do Extremo Sul Catarinense - UNESC; Grupo de Estudos e Pesquisa em Promoção da Saúde - GEPPS; Criciúma; SC; Brasil.

2 Universidade Federal de Pernambuco; Vitória de Santo Antão; Pernambuco; Brasil.

3 Faculdade do Vale do Araranguá (FVA)

4 Universidade do Extremo Sul Catarinense - UNESC; Programa de Pós-Graduação em Saúde Coletiva - PPGSCol do relacionado aos comportamentos individuais (alimentação, controle do estresse, relacionamentos, comportamento preventivo e atividade física $)^{1}$. Referente ao último comportamento, a Organização Mundial da Saúde (OMS) evidencia que a inatividade física está entre as quatro principais causas de mortalidade no mundo e um dos grandes problemas de saúde pública ${ }^{2}$, eleita pela OMS como um dos fatores 
de risco a serem enfrentados com prioridade em conjunto com fumo, alimentação inadequada e o consumo prejudicial de álcool ${ }^{3}$.

O baixo nível de atividade física pré-dispõe as pessoas ao adoecimento, consumo elevado de medicamento, a incidência relativa de doença arterial coronariana, infarto agudo do miocárdio, hipertensão arterial, câncer de cólon, câncer de mama, diabetes do tipo II e osteoporose, correspondendo a $63 \%$ de óbitos ocorridos no mundo em $2008^{3,4}$. O diabetes e a hipertensão constituem maior causa de hospitalização no Brasil ${ }^{5}$, seguido das doenças cardiovasculares, principal causa de mortalidade no país ${ }^{6}$, com um custo aproximado de 165 bilhões de reais para o Sistema Único de Saúde (SUS) 7 .

No Brasil, os gastos com medicamentos da atenção básica tiveram um aumento de $75 \%$ e com medicamentos dos programas estratégicos $124 \%$, de acordo com o Ministério da Saúde (MS) R\$ 35 bilhões estão sendo investidos na aquisição de medicamentos até 2016. Grande parte da população busca tratamento farmacológico para o controle de doenças, no entanto, o uso abusivo, insuficiente ou inadequado de medicamentos não beneficia a população e desperdiça os recursos públicos ${ }^{9}$ sobretudo no Brasil, cujo sistema de saúde, centrado nas ações de promoção vinculadas à Atenção Primária propõe a distribuição gratuita de medicamentos para uma série de doenças.

Gastos possivelmente evitados com a inserção de programas de atividades físicas para a população, os quais são considerados como estratégicos pelo MS para a prevenção e controle dessas doenças, pois evidências apontam que a atividade física regular tem sido uma forma não medicamentosa muito eficiente para prevenir e tratar diversas doenças, principalmente em relação às Doenças Crônicas Não Transmissíveis (DCNT) $)^{10,11}$. Portanto, aumentar os níveis de atividade física da população pré-dispõe da diminuição de eventos cardíacos, reduz a incidência de acidente vascular cerebral, hipertensão arterial, diabetes mellitus tipo II, câncer, doença vesicular, obesidade, depressão e ansiedade ${ }^{12,13}$ juntamente com melhora da habilidade motora, o desenvolvimento psicológico e o nível de relação social, contudo gera uma grande economia para o sistema de saúde ${ }^{14}$.

Compreendendo que, sobretudo o desenvolvimento de ações que promovam a saúde e sejam efetivas, necessitam necessariamente de análise do território, com diagnostico de hábitos, perfis e outros comportamentos, daquela realidade local. Neste estudo para além dos itens adscritos, observamos a necessidade de avaliar e relacionar o uso de medicamentos com o nível de atividade física da população pertencente ao distrito Sanitário de Santa Luzia do Município de Criciúma- SC, na perspectiva de adquirir informações para proposição de intervenções efetivas na promoção da atividade física em Unidades Básicas de Saúde.

\section{Metodologia}

Trata-se de um estudo descritivo por identificar, registrar e analisar as características da população, e transversal por avaliar a associação entre atividade física e consumo de medicamentos. A pesquisa teve aprovação no Comitê de Ética e Pesquisa em Seres Humanos sob o protocolo 379.255/2013.

A população foi composta por indivíduos de ambos os sexos, moradores Distrito Sanitário Santa Luzia e pertencentes as Estratégias Saúde da Família, totalizando 11 bairros. A amostra é representativa a quantidade total de moradores 12.325, totalizando 386 pessoas, para seleção dos participantes foi levado em consideração o número total de moradores e de ruas do bairro de acordo com a área geográfica, os entrevistados foram escolhidos por meio de sorteio aleatório tendo como critérios de inclusão estar na residência no momento da visita, preenchi- 
mento do TCLE e preenchimento completo dos instrumentos, para os adolescentes foi exigido à autorização dos pais ou responsáveis.

Todos os dados foram coletados por residentes de Educação Física do programa Residência em Saúde Coletiva da UNESC e acadêmicos do curso de Educação Física da mesma instituição, devidamente capacitados para avaliar, não foram registradas as recusas devido a seleção ser censitária.

A coleta de dados foi realizada entre o mês de outubro de 2013 a fevereiro de 2014 , nos períodos matutino e vespertino. Os instrumentos utilizados para a coleta de dados continham questões referente a identificação pessoal, medicamento (tipo, quantidade, tempo de uso), co-morbidades construídos a partir do interesse de pesquisa e avaliados por auto relato. O nível de atividade física habitual pelo instrumento desenvolvido por Russel R. Pate e traduzido por Nahas, a classificação econômica de acordo com as descrições ${ }^{15}$. O Índice de Massa Corporal (IMC) foi obtido pelo peso e estatura referida calculado de acordo com Martins e Petroski ${ }^{16}$.

A classificação da variável para os adultos segue orientação da Organização Mundial da Saúde: baixo peso (IMC até $\left.18,4 \mathrm{~kg} / \mathrm{m}^{2}\right)$, peso normal $(18,4-24,9 \mathrm{~kg} /$ $\left.\mathrm{m}^{2}\right)$, sobrepeso $\left(25,0-29,9 \mathrm{~kg} / \mathrm{m}^{2}\right)$, obesidades grau I $\left(30,0-34,9 \mathrm{~kg} / \mathrm{m}^{2}\right)$, grau II $\left(35,0-39,9 \mathrm{~kg} / \mathrm{m}^{2}\right)$ e grau III $\left(40,0 \mathrm{~kg} / \mathrm{m}^{2}\right.$ ou mais).

Para os adolescentes seguiu-se a classificação de Cole e colaboradore ${ }^{17}$ : peso normal (masculino até $21,8 \mathrm{~kg} / \mathrm{m}^{2}$ e feminino até $22,5 \mathrm{~kg} / \mathrm{m}^{2}$ ), sobrepeso (masculino de $21,9-23,3 \mathrm{~kg} / \mathrm{m}^{2}$ e feminino $22,6-24,4 \mathrm{~kg} / \mathrm{m}^{2}$ ) e obesidade (masculino $26,8-28,9 \mathrm{~kg} /$ $\mathrm{m}^{2}$ e feminino $\left.27,8-29,1 \mathrm{~kg} / \mathrm{m}^{2}\right)$. Os dados econômicos seguiram os critérios de classificação da $\mathrm{ABEP}^{15}$, subdivididos nas classes: A1, A2, B1, B2, C1, C2 e D, representando renda familiar de $\mathrm{R} \$ 895,00$ para classificação D a R $\$ 11.037,00$ para a classe A.

Os dados foram tabulados e categorizados no software Statistical Package for the Social Sciencies (SPSS) versão 20.0, sendo utilizada estatística descritiva com cálculo da frequência absoluta e relativa (percentual), da média e desvio padrão, mediana e intervalo interquartil. O teste de normalidade de Kolmogorov-Smirnov que apresentou distribuição normal para a variável total de medicamentos, o teste anova e Test t para media e desvio padrão, e nas demais variáveis o teste de Kruskal-Wallis e o teste U de Mann-Whitney para mediana e intervalo interquartil. A correlação utilizada foi de Pearson entre idade e total de medicamentos e de Spearman entre as demais variáveis.

\section{Resultados}

Na tabela 1, as variáveis estão expressas em Frequência Absoluta (F.A.), Frequência Relativa (\%) e nível de significância, $\mathrm{p}<0,01$ onde os valores de F. A. e \% são categorizados em sexo, idade, classificação econômica, IMC e nível de atividade física habitual e estratificados quanto aos grupos medicamentos e não medicamentos, mencionando o $p^{<0,01}$ para a quantidade de medicamentos utilizada em relação às categorias.

Para avaliar o consumo de medicamento total, para doenças do coração, hipertensão arterial, diabetes mellitus tipo II e outros (tabela 2), pelas variáveis: sexo, idade, perfil econômico, IMC e nível de atividade física habitual foram utilizadas duas análises: para comparação entre os sexos o teste $t$, e para as demais variáveis ANOVA oneway, ambas com significância de $\mathrm{p} \leq 0,05$, com IC de $95 \%$.

Na tabela 3 são apresentados os valores de correlação entre o total de medicamentos e idade, verificada através da correlação de Person. Para a correlação entre perfil socioeconômico, IMC e nível de atividade física habitual, foi utilizada a correlação de Sperman, com nível de significância de $\mathrm{p} \leq 0,05$, com IC de 95\%. 
TABELA 1 - Características sociodemográficas e comportamentais da amostra associadas ao uso de medicamentos. Criciúma, SC, Brasil $(n=386)$.

\begin{tabular}{|c|c|c|c|c|c|c|c|}
\hline \multirow{3}{*}{ VARIÁVIES } & \multirow{2}{*}{\multicolumn{2}{|c|}{$\begin{array}{c}\text { Característica Geral } \\
\qquad n=386\end{array}$}} & \multicolumn{2}{|c|}{ Medicamentos } & & \multicolumn{2}{|c|}{$\tilde{N}$ medicamentos } \\
\hline & & & \multirow{2}{*}{$\frac{n=212}{F . A}$} & \multicolumn{2}{|l|}{$54,92 \%$} & \multirow{2}{*}{$\frac{n=174}{F . A}$} & \multirow{2}{*}{$\frac{45,08 \%}{\%}$} \\
\hline & F.A & $\%$ & & $\%$ & $p$ & & \\
\hline \multicolumn{8}{|l|}{ SEXO } \\
\hline Feminino & 277 & 72,0 & 153 & 55,2 & \multirow{2}{*}{0,69} & 124 & 44,8 \\
\hline Masculino & 109 & 28,0 & 59 & 54,1 & & 50 & 45,9 \\
\hline \multicolumn{8}{|l|}{ IDADE } \\
\hline Adolescente & 32 & 8,2 & 4 & 25 & \multirow{4}{*}{0,00} & 28 & 87,5 \\
\hline Adulto jovem & 57 & 14,7 & 17 & 30,0 & & 40 & 70,1 \\
\hline Adulto & 207 & 53,6 & 122 & $58,9^{*}$ & & 85 & 41,1 \\
\hline Idoso & 90 & 23,3 & 70 & $77,8^{*}$ & & 20 & 22,2 \\
\hline \multicolumn{8}{|l|}{ ECONÔMICA } \\
\hline A1 & 2 & 0,5 & 1 & 50,0 & \multirow{7}{*}{0,01} & 1 & 50,0 \\
\hline A2 & 5 & 1,3 & 3 & 60,0 & & 2 & 40,0 \\
\hline B1 & 22 & 5,7 & 9 & 40,9 & & 13 & 59,1 \\
\hline B2 & 113 & 29,3 & 56 & 49,6 & & 57 & 50,4 \\
\hline C1 & 145 & 37,6 & 80 & 55,2 & & 65 & 44,8 \\
\hline C2 & 68 & 17,6 & 39 & 57,4 & & 29 & 42,6 \\
\hline D & 31 & 8,0 & 24 & $77,4^{*}$ & & 7 & 22,6 \\
\hline \multicolumn{8}{|l|}{ IMC } \\
\hline Baixo & 13 & 3,4 & 4 & 30,8 & \multirow{6}{*}{0,00} & 9 & 69,2 \\
\hline Peso normal & 124 & 32,1 & 49 & 39,5 & & 75 & 60,5 \\
\hline Sobrepeso & 131 & 33,9 & 74 & 56,5 & & 57 & 43,5 \\
\hline Obesidade I & 82 & 21,2 & 56 & 68,3 & & 26 & 31,7 \\
\hline Obesidade II & 27 & 7,0 & 21 & 77,8 & & 6 & 22,2 \\
\hline Obesidade III & 9 & 2,3 & 8 & $88,9^{*}$ & & 1 & 11,1 \\
\hline \multicolumn{8}{|c|}{ ATIVIDADE FÍSICA } \\
\hline Inativo & 169 & 43,8 & 101 & $59,8^{*}$ & \multirow{4}{*}{0,04} & 68 & 40,2 \\
\hline Mod. Ativo & 170 & 44,0 & 95 & 55,9 & & 75 & 44,1 \\
\hline Ativo & 41 & 10,6 & 15 & 36,6 & & 26 & 63,4 \\
\hline Muito ativo & 6 & 1,6 & 1 & 16,7 & & 5 & 83,3 \\
\hline
\end{tabular}

Fonte: dados da pesquisa. Legenda: IMC: Índice de Massa Corporal. F.A: Freqüência Absoluta; *significância entre as categorias $(p<0,01)$.

TABELA 2 - Comparação da média, desvio padrão, medianas e intervalo interquartil das variáveis relacionado a quantidade do uso de medicamentos conforme características sociodemográficas e comportamentais. Criciúma, SC, Brasil ( $n=386)$.

\begin{tabular}{|c|c|c|c|c|c|c|c|c|c|c|}
\hline \multirow{2}{*}{ VARIÁVEIS } & \multicolumn{2}{|c|}{ Consumo Geral } & \multicolumn{2}{|l|}{ Coração } & \multicolumn{2}{|c|}{ Hipertensão } & \multicolumn{2}{|l|}{ Diabetes } & \multicolumn{2}{|l|}{ Outros } \\
\hline & Média (DP) & $p$ & Mediana (i i) & $p$ & Mediana (i i) & $p$ & Mediana (i i) & $p$ & Mediana (i i) & $p$ \\
\hline \multicolumn{11}{|l|}{ SEXO } \\
\hline Feminino & $2,11( \pm 1,53)$ & \multirow{2}{*}{0,15} & $1,00(1,00-2,00)$ & \multirow{2}{*}{0,43} & $0,56(1,00-2,00)$ & \multirow{2}{*}{0,35} & $1,00(1,00-1,00)$ & \multirow{2}{*}{0,31} & $1,00(1,00-2,00)$ & \multirow{2}{*}{0,77} \\
\hline Masculino & $2,44( \pm 1,34)$ & & $1,00(1,00-2,00)$ & & $1,00(1,00-1,75)$ & & $1,00(1,00-2,00)$ & & $1,00(1,00-2,00)$ & \\
\hline \multicolumn{11}{|l|}{ IDADE } \\
\hline Jovem & $1,50( \pm 0,70)$ & \multirow{4}{*}{0,05} & - & & - & & - & & $1,50(1,00)$ & \\
\hline Adulto jovem & $1,25( \pm 0,48)^{*}$ & & - & & $1,00(1,00)$ & & $1,00(1,00-1,00)$ & & $1,00(1,00-1,00)$ & \\
\hline Adulto & $2,17( \pm 1,55)$ & & $1,00(1,00-2,00)$ & & $1,00(1,00-2,00)$ & & $1,00(1,00-2,00)$ & & $1,00(1,00-2,00)$ & \\
\hline Idoso & $2,53( \pm 1,45)^{*}$ & & $1,00(1,00-2,00)$ & & $1,00(1,00-2,00)$ & & $1,00(1,00-1,75)$ & & $1,00(1,00-2,00)$ & \\
\hline
\end{tabular}


... continua

SOCIOECONÔMICO

\begin{tabular}{|c|c|c|c|c|c|c|c|c|c|}
\hline A1 & $1,00(-)$ & \multirow{7}{*}{0,78} & - & \multirow{7}{*}{0,19} & - & \multirow{7}{*}{0,18} & - & \multirow{7}{*}{0,4} & \\
\hline A2 & $2,00( \pm 1,00)$ & & - & & $1,00(1,00-1,00)$ & & $1,00(1,00-1,00)$ & & $1,00(1,00-1,00)$ \\
\hline B1 & $2,55( \pm 1,81)$ & & $1,00(1,00-1,00)$ & & $1,00(1,00-2,00)$ & & $1,00(1,00-1,00)$ & & $2,00(1,00-)$ \\
\hline B2 & $2,26( \pm 1,38)$ & & $2,00(1,00-2,00)$ & & $1,00(1,00-2,00)$ & & $1,00(1,00-2,00)$ & & $1,00(1,00-1,00)$ \\
\hline $\mathrm{C} 1$ & $2,08( \pm 1,12)$ & & $1,00(1,00-1,00)$ & & $1,00(1,00-2,00)$ & & $1,00(1,00-1,00)$ & & $1,00(1,00-2,00)$ \\
\hline$C 2$ & $2,10( \pm 1,81)$ & & $1,00(1,00-1,75)$ & & $1,00(1,00-1,00)$ & & $1,00(1,00-2,00)$ & & $1,00(1,00-2,00)$ \\
\hline D & $2,54( \pm 2,08)$ & & $1,50(1,00-2,25)$ & & $1,50(1,00-2,00)$ & & $1,00(1,00-1,50)$ & & $1,00(1,00-2,00)$ \\
\hline \multicolumn{10}{|l|}{ IMC } \\
\hline Baixo peso & $1,75( \pm 0,96)$ & \multirow{6}{*}{0,3} & $1,00(1,00-1,00)$ & & $2,00(2,00-2,00)$ & \multirow{6}{*}{0,43} & $1,00(1,00-1,00)$ & \multirow{6}{*}{0,85} & $1,50(1,00-1,50)$ \\
\hline Peso normal & $2,06( \pm 1,62)$ & & $1,00(1,00-2,00)$ & \multirow{5}{*}{0,79} & $1,00(1,00-2,00)$ & & $1,00(1,00-1,00)$ & & $1,00(1,00-2,00)$ \\
\hline Sobrepeso & $2,02( \pm 1,36)$ & & $1,00(1,00-2,00)$ & & $1,00(1,00-1,50)$ & & $1,00(1,00-1,00)$ & & $1,00(1,00-2,00)$ \\
\hline Obesidade I & $2,51( \pm 1,45)$ & & $1,50(1,00-2,00)$ & & $1,00(1,00-2,00)$ & & $1,00(1,00-1,75)$ & & $1,00(1,00-2,00)$ \\
\hline Obesidade II & $2,14( \pm 1,31)$ & & $1,00(1,00-1,00)$ & & $1,00(1,00-1,25)$ & & $1,00(1,00-1,75)$ & & $1,00(1,00-2,00)$ \\
\hline Obesidade III & $2,87( \pm 2,29)$ & & $1,50(1,00)$ & & $1,00(1,00-2,00)$ & & $1,00(1,00)$ & & $1,00(1,00)$ \\
\hline \multicolumn{10}{|c|}{ ATIVIDADE FÍSICA } \\
\hline Inativo & $2,47( \pm 1,67)^{\#}$ & \multirow{4}{*}{0,05} & $1,00(1,00-1,00)$ & \multirow{4}{*}{0,19} & $1,00(1,00-2,00)$ & \multirow{4}{*}{0,55} & $1,00(1,00-1,50)$ & \multirow{4}{*}{0,62} & $1,00(1,00-2,00)$ \\
\hline Mod. Ativo & $1,98( \pm 1,28)$ & & $1,00(1,00-2,00)$ & & $1,00(1,00-2,00)$ & & $1,00(1,00-1,00)$ & & $1,00(1,00-1,25)$ \\
\hline Ativo & $1,80( \pm 1.08)^{\#}$ & & $2,00(1,00)$ & & $1,00(1,00-2,00)$ & & $1,00(1,00-1,00)$ & & $1,00(1,00-1,00)$ \\
\hline Muito ativo & $2(-)$ & & - & & - & & - & & $2,00(2,00-2,00)$ \\
\hline
\end{tabular}

Fonte: dados da pesquisa. Legenda: ii=Intervalo Interquartil * comparação adulto jovens com idosos; \#comparação de inativos com ativos $(p<0,05)$.

TABELA 3 - Correlação entre as variáveis sociodemográficas e comportamentais com uso de medicamentos. Criciúma, SC, Brasil ( $n=386)$.

\begin{tabular}{|c|c|c|c|c|c|c|c|c|c|c|}
\hline \multirow{2}{*}{ VARIÁVEIS } & \multicolumn{2}{|c|}{ Total Geral } & \multicolumn{2}{|c|}{ Coração } & \multicolumn{2}{|c|}{ Hipertensão } & \multicolumn{2}{|c|}{ Diabetes } & \multicolumn{2}{|c|}{ Outros } \\
\hline & rs & P & is & P & is & P & rs & P & rs & P \\
\hline Idade & $0,22 \mathrm{a}$ & $0,00^{*}$ & $-0,17$ & 0,27 & 0,00 & 0,97 & 0,00 & 1,00 & 0,05 & 0,58 \\
\hline Socioeconômico & $-0,07$ & 0,32 & $-0,02$ & 0,90 & 0,05 & 0,61 & $-0,03$ & 0,83 & 0,11 & 0,23 \\
\hline IMC & $0,16 b$ & 0,02 & 0,04 & 0,78 & 0,03 & 0,72 & 0,20 & 0,17 & $-0,03$ & 0,73 \\
\hline Atividade Física & $0,17^{*}$ & 0,01 & 0,25 & 0,11 & $-0,09$ & 0,31 & $-0,11$ & 0,46 & $-0,12$ & 0,19 \\
\hline
\end{tabular}

Fonte: dados da pesquisa. Legenda: a=correlação Pearson, entre idade e consumo total de medicamentos ( $p<0,05), b=$ correlação entre IMC com o consumo total de medicamentos $(p<0,05)$ e Estilo de Vida com o consumo total de medicamentos ${ }^{*}$ rs =correlação de Sperman.

\section{Discussão}

Ao avaliar a prevalência do uso de medicamentos pelos participantes do estudo os resultados demonstraram que $54,92 \%$ dos participantes da pesquisa fazem uso crônico de algum tipo de medicação, resultado semelhante ao encontrado no município de Pelotas-RS ${ }^{18}$. Da mesma forma, a maior participação nestes estudos é do sexo feminino (72\%) sendo justificado pelo horário das entrevistas (matutino e vespertino), pois influenciadas pela cultura e organização social em que vivem, na maioria das vezes elas são as responsáveis pelas tarefas domésticas ${ }^{19,20}$.

Quanto ao uso contínuo de medicamentos, $55,2 \%$ das mulheres fazem uso, semelhante ao encontrado por Costa e colaboradores ${ }^{21}$ em que o sexo feminino foi relacionado ao maior uso de medicamentos. Quando referenciada a quantidade de medicamentos utilizada, a média foi de $2,44( \pm 1,34)$ e $2,11( \pm 1,53)$ unidades de medicamentos para homens e mulheres respectivamente, quantidades mais elevadas quando comparado com o estudo realizado na cidade de Campinas $(\mathrm{SP})^{21} \mathrm{com}$ um consumo médio de 1,8 medicamentos. 
Justifica-se relacionar a idade e o consumo de medicamentos devido à incidência de DCNT apresentar aumento com o avanço da idade, que por consequência pode haver o aumento no consumo de medicamento em ambos os sexos ${ }^{21}$. Neste estudo foram observadas diferenças significativas no uso de medicamentos relacionado a idade $(\mathrm{p}<0,05)$, com uma prevalência de 77,8\% em idosos e 58,9\% em adultos. Os idosos também apresentaram maior consumo em relação a quantidade de medicamentos, com média de $2,53( \pm 1,45)$ unidades de medicamentos, encontrando correlações positivas $(r=0,22)$ entre idade e consumo com nível de significância de $\mathrm{p} \leq 0,01$, similares a outros estudos ${ }^{21,22}$.

$\mathrm{Na}$ relação consumo de medicamento e classificação socioeconômica foram encontradas divergências na literatura, onde Bertoldi e colaboradores ${ }^{22}$ apontam relação direta entre consumo de medicamento e perfil socioeconômico, enquanto Silva e colaboradores ${ }^{4}$ descrevem que o perfil socioeconômico não teve associação com o consumo de medicamentos. Em nossos achados 66,9\% da amostra era das classes B2 e C1 (renda familiar de 2 a 4 salários mínimos) ${ }^{15}$, e referente ao consumo de medicamento a classe $\mathrm{D}$ demonstrou maior incidência com $77,4 \%$ de indivíduos, apresentando diferença significativa quando relacionado as demais classes $(\mathrm{p}<0,01)$. Quando comparadas as médias pressupõe uma diferença no consumo de medicamentos entre as classes econômicas, A2 2,00( $\pm 1,00)$ e D 2,54( $\pm 2,08)$, no entanto sem apresentar correlações significativas.

$\mathrm{Na}$ a análise do IMC da amostra 33,9\% encontra-se com sobrepeso e 30,5\% como obesos (grau I, II e III). Na relação do IMC com o consumo de medicamento, observa-se que $88,9 \%$ dos obesos (grau III) e 77,8\% (grau II) utilizam medicamentos, com resultados significativamente diferentes $(\mathrm{p} \leq 0,05)$, onde $60,5 \%$ dos indivíduos com peso normal não fazem uso contínuo de medicamentos. Neste grupo de obesos também foram encontrados o maior consumo de fármacos $(2,87 \pm 2,29)$ com maior prevalência para remédios relacionados a doenças cardiovasculares. Embora não tenham apresentado significância na comparação das médias foi observada uma correlação positiva $(r=0,16 ; \mathrm{p}<0,02)$ entre a categoria o aumento do IMC e do uso de medicamentos. Portanto, os comportamentos inadequados à saúde (excesso de peso, alimentação inadequada, uso de fumo e álcool, inatividade física) aumentam as chances de desenvolvimento de DCNT e dessa forma, eleva-se o consumo de medicamentos, agravando este problema para a saúde pública ${ }^{1,2,21}$.

Compreendendo a prática de atividade física habitual como um dos fatores que compões o estilo de vida ${ }^{1}$, na população estudada apenas $10,6 \%$ foram considerados ativos com $43,8 \%$ de inativos, resultados semelhantes ao estudo realizado por Hallal e colaboradores ${ }^{23}$. Relacionando o nível de atividade física com o consumo de medicamentos, 59,8\% dos inativos utilizam medicamentos apresentou diferença significativa na análise com os demais grupos $(\mathrm{p}<0,04)$. Conforme aumenta a atividade física habitual, reduz o uso de medicamentos, podendo observar altas prevalência de indivíduos que não utilizam medicamento nos grupos moderadamente ativo $(44,1 \%)$, ativo $(63,4 \%)$ e muito ativo $(83,3 \%)$.

Essa relação direta entre o nível de atividade física e consumo de medicamento corrobora com o estudo de Bertoldi e colaboradores ${ }^{22}$, sendo encontrado que o consumo de medicamento dos ativos $1,80( \pm 1.08)$ foi significativamente $(\mathrm{p} \leq 0,01)$ menor que a média de inativos $2,47( \pm 1,67)$. O uso de medicamentos foi menor ao encontrado por Silva e colaboradores ${ }^{4}$ onde a proporção do uso dos sujeitos insuficientemente ativos foi de quatro ou mais medicamentos.

Observando a correlação do nível de atividade física e o uso de medicamentos foi verificado significância $(\mathrm{r}=0,17 ; 0,01)$, apresentando uma proporção maior de uso de 
medicamentos conforme a inatividade física do indivíduo, respostas semelhantes as encontradas nas pesquisas ${ }^{4,22}$. Todos os achados nos remetem a pensar em estratégias de cuidado das pessoas, considerando para além do estilo de vida outros comportamentos bem como o território onde vivem, para então propor intervenções efetivas.

A partir deste estudo verifica-se a necessidade de programas ou estratégias para atendimento a saúde do homem, tendo em vista que os homens apresentaram um consumo maior de medicamento comparado as mulheres, e considerando as dificuldades que o SUS tem em desenvolver ações específicas para esta população, tem-se como indicativo problemas maiores em um futuro próximo.

Uma das estratégias para este cuidado é o tratamento não farmacológico, estimulando não somente os homens, mas todas as pessoas a prática regular de atividade física. As pessoas fisicamente ativas diminuem o consumo de medicamentos e consequentemente os gastos com fármacos tendem a diminuir, podendo ser revertido em programas de promoção da saúde e qualidade de vida. A população das classes econômicas menores também apresentou consumo maior de medicamento, apontando a necessidade de estratégias para mais específicas, como uma dispensação adequada, e orientações sobre o consumo consciente.

Portanto se faz necessário a incorporação do profissional de Educação Física junto as Unidades Básicas de Saúde e das Estratégias de Saúde da Família, na construção de ações para uma mudança no estilo de vida, favorecendo a diminuição dos gastos públicos na dispensação de medicamento, tratamentos e hospitalizações.

\section{Financiamento}

Este estudo foi desenvolvido com financiamento próprio.

\section{Referências}

1. Nahas MV. Atividade física, saúde e qualidade de vida. $6^{\circ} \mathrm{ed}$. Londrina: Madiograf; 2013. p. 24-25.

2. World health organization. Global health risks: mortality and burden of disease attributable to selected major risks. Geneva: World Health Organization; 2009. Disponível em: http://www.who.int/healthinfo/global_burden_disease/GlobalHealthRisks_ report_full.pdf (acessado em 16 de Abril de 2015)

3. Duncani BB , et al. Doenças Crônicas Não Transmissíveis no Brasil: prioridade para enfrentamento e investigação. Revista de Saúde Pública. 2012; 126-134.

4. Silva LJ, et al. Association between levels of physical activity and use of medication among older women. Caderno de Saúde Pública. Rio de Janeiro. 2012; 28(3):463-471.

5. Brasil. Ministério da Saúde. Secretaria de Vigilância em Saúde. Departamento de Análise de Situação de Saúde. Plano de ações estratégicas para o enfrentamento das doenças crônicas não transmissíveis (DCNT) no Brasil 2011-2022. Ministério da Saúde. Secretaria de Vigilância em Saúde. Departamento de Análise de Situação de Saúde. Brasília: Ministério da saúde; 2011. Disponível em: http://bvsms.saude.gov.br/bvs/publicacoes/ plano_acoes_enfrent_dcnt_2011.pdf (acessado em 16 de Abril de 2015)

6. Goulart FA. Doenças crônicas não transmissíveis: estratégias de controle e desafios e para o os sistemas de saúde. Brasília. Organização PanAmericana da Saúde; [2011] acesso em 2015 abril 16]. Disponível em: <http://apsredes.org/site2012/wp-content/ uploads/2012/06/Condicoes-Cronicas_flavio1.pdf

7. Brandão AA, et al. Hipertensão. $2^{\circ}$ ed. Rio de Janeiro: Elsevier. 2012.

8. Vieira FS. Gasto do Ministério da Saúde com medicamentos: tendência dos programas de 2002 a 2007. Revista de Saúde Pública. 2009; 43 (4):674-681.

9. Brasil. Ministério da Saúde. Secretaria de Vigilância em Saúde. Vigitel 2012: Vigilância de fatores de risco e proteção para doenças crônicas por inquérito telefônico. Brasília: Ministério da Saúde; 2013. 
10. Ciolac EG, Guimarães GV. Exercício físico e síndrome metabólica. Revista Brasileira de Medicina do Esporte. São Paulo. 2004; 10 (4): 319-24.

11. Samitz G, et al. Domains of physical activity and all-cause mortality: systematic review and dose-response meta-analysis of cohort studies. International Journal of Epidemiology. 2011; 40:1382-400.

12. American college of sports medicine. Diretrizes do ACSM para o teste de esforço e sua prescrição. $7^{\circ}$ ed. Rio de Janeiro: Guanabara Koogan; 2007.

13. Giraldo AED, et al. Influência de um programa de exercícios físicos no uso de serviços de saúde na Atenção Básica de Saúde do município de Rio Claro, SP. Revista Brasileira de Atividade Física e Saúde. Pelotas. 2013; 2 (18): 186-196.

14. Florindo AA, et al. Practice of physical activities and associated factors in adults, Brazil, 2006. Revista Saúde Pública. 2009; 43(S2): 65-73.

15. Associação brasileira de empresas de pesquisa-ABEP. Dados com base no Levantamento Sócio Econômico. São Paulo: 2012.

16. Martins MO, Petroski EL. Revista Brasileira de Cineantropometria e Desempenho Humano, Florianópolis. 2000; 58-65.

17. Cole TIM, et al. Establishing a standard definition for child overweight and obesity worldwide: international survey. British Medical Journal. Londres. 2000; 320: 1-6.

18. Bertoldi AD, et al. Physical activity and medicine use: evidence from a population-based study. BMC Public Health. 2006; 6 (224):1-6.

19. Barros MV, Nahas MV. Comportamento de risco, auto-avaliação do nível de saúde e percepção de estresse entre trabalhadores de indústria. Revista de Saúde Pública. 2001; 35: 554-63.

20. World health organization. Shaping the Future. Gender, health and aging. The World health report. Geneva: World Health Organization; Outubro de 2003. Disponível em: http://www.who.int/whr/2003/en/overview_en.pdf (acessado em 16 de Abril de 2015)

21. Costa KS, et al. Utilização de medicamentos e fatores associados: um estudo de base populacional no Município de Campinas, São Paulo, Brasil. Caderno de Saúde Pública. 2011; 27(4): 649-658.

22. Bertoldi AD, et al. Utilização de medicamentos em adultos: prevalência e determinantes individuais. Revista de Saúde Pública. 2004; 38 (2): 228-38.

23. Hallal PC, et al. Global physical activity levels: surveillance progress, pitfalls, and prospects. Lancet. 2012; 380:247-57.

ENDEREÇO PARA

CORRESPONDÊNCIA

JONI MARCIO DE FARIAS

jmf@unesc.net
Universidade do Extremo Sul Catarinense

- UNESC; Programa de Pós-Graduação em

Saúde Coletiva - PPGSCol; Grupo de Estudos

e Pesquisa em Promoção da Saúde - GEPPS;

Criciúma; SC; Brasil; Rua Madre Tereza Michel

$n^{\circ} 327$, apto 202; CEP 88803-030
RECEBIDO 19/11/2015

REVISADO 25/04/2016

$29 / 04 / 2016$

$03 / 05 / 2016$

05/05/2016

APROVADO 08/07/2016 